\title{
Interfacial synthesis of polyethyleneimine-protected copper nanoclusters: Size-dependent tunable photoluminescence, pH sensor and bioimaging
}

\author{
Chan Wang ${ }^{\mathrm{a}}$, Yagang Yao ${ }^{* \mathrm{~b}}$, Qijun Song ${ }^{{ }^{\mathrm{a}}}$
}

${ }^{a}$ Key Laboratory of Food Colloids and Biotechnology, Ministry of Education, School of Chemical \& Material Engineering, Jiangnan University, Wuxi 214122, China.

${ }^{\mathrm{b}}$ Division of Advanced Nanomaterials, Key Lab of Nanodevices and Applications, Suzhou Institute of Nano-tech and Nano-bionics, Chinese Academy of Sciences, University of Chinese Academy of Sciences, Suzhou 215123, China.

\footnotetext{
* ${ }^{\mathrm{b}}$ Corresponding author. Tel.: +86-512-62872829; E-mail addresses: ygyao2013@ sinano.ac.cn. (Y. Yao).

*a Corresponding author. Tel.: +86-510-85917763; E-mail addresses: qsong@jiangnan.edu.cn (Q. Song).
} 


\begin{abstract}
The copper nanoclusters (CuNCs) offer excellent potential as functional biological probes due to their unique photoluminescence (PL) properties. Herein, CuNCs capped with hyperbranched polyethylenimine (PEI) were prepared by the interfacial etching approach. The resultant PEI-CuNCs exhibited good dispersion and strong fluorescence with high quantum yields (QYs, up to 7.5\%), which would be endowed for bioimaging system. By changing the reaction temperatures from 25 to $150{ }^{\circ} \mathrm{C}$, the size of PEI-CuNCs changed from 1.8 to $3.5 \mathrm{~nm}$, and thus tunable PL were achieved, which was confirmed by transmission electron microscopy (TEM) imagings and PL spectra. Besides, PEI-CuNCs had smart absorption characteristics that the color changes from colorless to blue with changing the $\mathrm{pH}$ value from 2.0 to 13.2, and thus they could be used as color indicator for $\mathrm{pH}$ detection. In addition, the PEI-CuNCs exhibited good biocompatibility and low cytotoxicity to $293 \mathrm{~T}$ cells through MTT assay. Owing to the positively charged of PEI-CuNCs surface, they had the ability to capture DNA, and the PEI-CuNCs/DNA complexes could get access to cells for efficient gene expression. Armed with these attractive properties, the synthesized PEI-CuNCs are quite promising in biological applications.
\end{abstract}

Keywords: interfacial synthesis, copper nanoclusters, tunable photoluminescence, $\mathrm{pH}$ sensor, bioimaging 


\section{Introduction}

Metal nanoclusters (NCs) composing of several to tens of atoms bridge the gap between traditional organometallic compounds and crystalline metal nanoparticles (NPs), and exhibit molecule-like properties, such as HOMO-LUMO transition, molecular magnetism, and strong luminescence [1-4]. Due to their tunable fluorescence, high photoluminescence (PL) quantum yields and facile synthesis process [5-8], metal NCs have recently attracted increased interest $[9,10]$, which are endowed the potential applications in catalysis, chemical sensors and electronic devices [11,12]. Particularly, the low toxicity, good biocompatibility and multifunctional surface chemistry make metal NCs an ideal candidate for biological imaging [13-16]. Compared with $\mathrm{Au}, \mathrm{Ag}$ and $\mathrm{Ag} / \mathrm{Au}$ alloy nanomaterials [17-19], non-precious copper is earth-abundant, extremely cheap and widely used in daily life, and the corresponding CuNCs own unique PL properties [20,21], but they still suffer from the difficulties in controlling the tiny size and the stability in an aqueous medium.

Normally, the fluorescent CuNCs were synthesized via the bottom-up or top-down approaches [22-25]. The bottom-up synthesis was mainly based on the chemical reduction of copper ions using sodium ascorbate or $\mathrm{N}_{2} \mathrm{H}_{4} \cdot 2 \mathrm{H}_{2} \mathrm{O}$ as the reducing agent, while the suitable capping agents were usually needed to etch the larger CuNPs down to smaller CuNCs through the top-down synthesis [25]. Unfortunately, the as-prepared CuNCs by above two approaches tended to emit the fluorescence only in blue or red region [26-28]. It is still a challenge to synthesize CuNCs with tunable luminescence, which can be used as optical materials in light-emitting device (LED), biological detection and biolabeling [29-32]. Hence, it is highly desirable to develop a general and effective approach for the preparation of CuNCs with tunable PL.

Interfacial synthesis has attracted much attentions as a promising method for synthesis and assembly of nanomaterials [33], such as semiconductor NPs, two-dimensional superlattices and 3D structures [34-36]. Interfacial phenomena are complex while common, which involves the interfacial potential, surface tension, concentration gradients, etc. By optimizing these factors, the structure and properties of nanomaterials could be controlled. Recently, Pradeep's group reported the synthesis of luminescent AgNCs and AuNCs by the interfacial route [37,38]. Wang et al. 
demonstrated the well-controlled production of organic-soluble fluorescent AuNCs using the interfacial etching of AuNPs [39]. However, methodologies to produce the fluorescent CuNCs by interfacial etching approach have never been studied nor understood.

Herein, we reported the synthesis of fluorescent CuNCs, PEI-CuNCs, through the interfacial etching of polyethyleneimine (PEI) protected copper nanoparticles (CuNPs) at the water-chloroform interface. Intriguingly, the resultant CuNCs exhibited strong, and size-dependent tunable PL. Moreover, the protection of PEI layer allowed CuNCs to have good dispersion in aqueous solution and showed the pH-responsive fluorescence. Confocal laser scanning microscopy studies using 293T cells as a model cell demonstrated that the PEI-CuNCs had been internalized in cell cytoplasm with good biocompatibility and low cytotoxicity. Further agarose gel electrophoresis assay revealed that the CuNCs have the ability to capture DNA. These advantages illustrated the promising applications of PEI-CuNCs in the gene delivery and bioimaging.

\section{Experimental}

\subsection{Materials}

Oleyl amine (OA), 1-octadecene (ODE), polyethylenimine (PEI) (MW=1800, 10000, $25000 \mathrm{~g} / \mathrm{mol}, 99 \%)$ and 3-[4,5-dimethylthiazol-2yl]-2,5-diphenyltetrazolium bromide (MTT) were purchased from Aldrich. Copper nitrate $\left(\mathrm{Cu}\left(\mathrm{NO}_{3}\right)_{2}\right)$, methanol, ethanol, formaldehyde $(\mathrm{HCHO}, 35 \%)$ and $\mathrm{CHCl}_{3}$ were analytical grade and used as received. 293T cells were available in the Cell Bank of Type Culture Collection of Chinese academy of sciences. Cell culture products and reagent, unless mentioned otherwise, were purchased from GIBCO. All reagents were used as received without further purification. In our experiment, the $\mathrm{pH}$ of Britton-Robinson (BR) buffers were adjusted to $2.0-13.2$ by adding $\mathrm{NaOH}$. Deionized water was used in all experiments.

\subsection{Preparation of the OA stabilized CuNPs}

The preparation of CuNPs were similar to the previously reported synthetic procedure with a slight modification [40]. Typically, $0.1 \mathrm{~g} \mathrm{Cu}\left(\mathrm{NO}_{3}\right)_{2} \cdot 6 \mathrm{H}_{2} \mathrm{O}$ was added into $15 \mathrm{~mL} \mathrm{OA}$ and $15 \mathrm{~mL}$ ODE. The resulting solution was slowly heated to $240{ }^{\circ} \mathrm{C}$ and maintained at this temperature for $15 \mathrm{~min}$ with stirring. The reaction was completed when the color changed from blue to brown. The resultant OA-CuNPs were precipitated by addition of $\mathrm{CH}_{3} \mathrm{OH}$, collected through centrifugation (5000 
$\mathrm{r} / \mathrm{min}$ ), and then re-dispersed in $\mathrm{CHCl}_{3}$ with their molarity as $25 \mathrm{mM}$.

\subsection{Interfacial Synthesis of PEI-protected CuNCs}

Typically, the interface of water-chloroform was formed by adding $3 \mathrm{~mL}$ aqueous solution (including $1 \mathrm{~mL}$ PEI and $0.5 \mathrm{~mL} \mathrm{HCHO}$ ) and $3 \mathrm{~mL} \mathrm{OA-CuNPs}$ in $\mathrm{CHCl}_{3}$. The etching reaction occurred at $50{ }^{\circ} \mathrm{C}$ with stirring for $4 \mathrm{~h}$, then the CuNCs were formed in aqueous phase under the protection of PEI. After standing for a certain time, the solution was stratified, and the oil phase was removed. The PEI-CuNCs in aqueous phase were concentrated by distillation under vacuum, followed by re-dispersed in water for subsequent study. For comparison, the PEI-CuNCs with different etching temperatures were also prepared with a similar procedure.

\subsection{Cellular imaging}

$293 \mathrm{~T}$ cells $\left(2^{10}\right.$ cells $\left./ \mathrm{mL}\right)$ were cultured in Dulbecco's modified Eagle's medium supplemented with $10 \%$ fetal bovine serum and $1 \%$ penicil-lin/streptomycin (DMEM) using 96-well plate. Suspensions of PEI-CuNCs from the stock solution were prepared with Dulbecco's phosphate buffer saline (DPBS). After sonication for 10 min to ensure complete dispersion, an aliquot (typically $0.01 \mathrm{~mL}$ ) of the suspension was added to the well of a chamber slide containing the cells cultured for $12 \mathrm{~h}$. The chamber slide was then incubated at $37{ }^{\circ} \mathrm{C}$ in a $\mathrm{CO}_{2}$ incubator for $12 \mathrm{~h}$ for PEI-CuNCs uptake, and the concentration of PEI-CuNCs is $20 \mu \mathrm{g} / \mathrm{mL}$ (only $10 \mu \mathrm{g}$ of PEI-CuNCs to $500 \mu \mathrm{L}$ of culture medium ( $10^{5}$ cells $)$ was added). Prior to fixation of the cells on the slide for inspection with a confocal fluorescence microscope, the excess PEI-CuNCs were removed by washing three times with DPBS.

\subsection{Gel retardation assay and plasmid DNA protection}

Electrophoresis was carried out in $1 \%(\mathrm{w} / \mathrm{v})$ agarose gel in $1 \times \mathrm{TAE}$ buffer at $90 \mathrm{~V}$ for $30 \mathrm{~min}$. The gels were stained with ethidium bromide (EtBr). Complexes for this assay were prepared at PEI-CuNCs: pDNA ratio (w/w, \%) of 5, 10, 15, 20, 25, 30, 35, 40 and 45 . The amount of pDNA $(0.5 \mathrm{mg})$ was constant.

\subsection{Characterization Methods}

UV-Vis absorption spectra were obtained by using ESCALAB $250 \mathrm{UV}$-Vis spectrophotometer. The PL experiments were performed with a Shimadzu RF-5301 PC spectrofluorimeter. X-ray photoelectron spectroscopy (XPS) using Mg $\mathrm{K} \alpha$ excitation (1253.6 eV) was collected in a VG ES-CALAB MKII spectrometer. Binding energy calibration was based on C 1 s at $284.6 \mathrm{eV}$. Powder X-ray diffraction 
(XRD) was carried out using a Siemens D5005 diffract meter using $\mathrm{Cu} K \alpha(1.5418 \AA$ ) radiation at room temperature. The Fourier transform infrared spectroscopy (FT-IR) was measured at wavenumbers ranging from $500 \mathrm{~cm}^{-1}$ to $4000 \mathrm{~cm}^{-1}$ using a FALA 2000-104 FT-IR spectrophotometer. The morphology and mean diameter of the resultant PEI-CuNCs were characterized by JEM-2100F transmission electron microscope (TEM) operating at $200 \mathrm{kV}$. The confocal microscopy images were taken at Olympus Fluoview FV1000 equipped by $2 \mathrm{~mW}$ laser power. The $\mathrm{pH}$ values of solutions were measured with a $\mathrm{pH}$ meter (PHS-3C, Shanghai Leici Instrument Company, Ltd., China). Zeta potential was measured by using a Zetasizer Nano Z (Malvern Instruments, Malvern, UK). Fluorescent lifetime measurements was done on Edinburgh Instruments F980.

\section{Results and discussion}

\subsection{Synthesis of CuNCs}

A schematic of the interfacial etching of CuNPs with PEI at the water-chloroform interface for synthesis of CuNCs was shown in Scheme 1. The procedure includes two steps: the synthesis of larger CuNPs, and the conversion of CuNPs into smaller CuNCs. The CuNPs were prepared through the reduction of $\mathrm{Cu}^{2+}$ ions using oleyl amine (OA) as both the reducing agent and stabilizer [40]. The OA was a common reducing agent for synthesizing nanomaterials, and OA-stabilized nanomaterials could be easily dispersed in organic solvents, such as toluene and chloroform [41]. In present work, the preformed OA-CuNPs were dispersed in chloroform. The second step occurred at the oil-water interface by etching of the preformed CuNPs with PEI, which is a water-soluble functional polycation polymer with the capability to etch the previously formed NPs to yield the NCs and as stabilizer on the surface of nanomaterials $[42,43]$. The resultant CuNCs in aqueous solution displayed strong fluorescence.

\subsection{Characterization of CuNCs}

To directly view the preformed CuNPs, the transmission electron microscope (TEM) analysis was performed, and the result is shown in Fig. 1a and b. As can be seen, the CuNPs are highly dispersible and exhibit spherical-shaped with an average size of $\sim 18 \mathrm{~nm}$. The X-ray diffraction (XRD) result of CuNPs is shown in Fig. 1c, and the peaks appear in the $2 \theta$ range from $30^{\circ}$ to $60^{\circ}$, which is the characteristic XRD 
response of the (111) and (200) planes, respectively [40]. After the etching process, the size decreases to $1.5-2.5 \mathrm{~nm}$ with the average size of $2.0 \mathrm{~nm}$, as displayed in Fig. 1d and e. The crystal lattice fringes are $2.05 \AA$ apart which indicates the (111) planes of the metallic $\mathrm{Cu}$ (see the inset of Fig. 1d). The result clearly demonstrated the larger CuNPs had been successfully etched to smaller CuNCs. Moreover, the resultant PEI-CuNCs exhibited good dispersion with no aggregation due to the protection of PEI [44]. The XRD result demonstrates that the characteristic diffraction peaks of CuNPs disappeared, suggesting the formation of the small CuNCs (Fig. 1f). The metal NCs were too small to possess all of the characteristic diffraction peaks of bulk NPs. Similar results had also been observed for previously reported metal NCs $[45,46]$.

Fig. 2a illustrates the UV-Vis absorption spectrum of CuNPs, and a broad absorbance band centered at $450 \mathrm{~nm}$ with a bandwidth of about $70 \mathrm{~nm}$. After the interfacial etching, the characteristic absorption bands of CuNPs disappeared, and a new strong plasma absorption with a maximum at about $300 \mathrm{~nm}$ appeared in the UV region, suggesting the decomposition of the CuNPs and the generation of small CuNCs in the etching process [27]. These results confirmed the difference between the molecule-like property, strong fluorescence, of metal NCs with that of the large metal NPs. After etching, the PEI-CuNCs in the aqueous solution exhibited strong fluorescence with their excitation and emission maxima located at 380 and $495 \mathrm{~nm}$, respectively (seen in Fig. 2b). Similar with previously reported CuNCs, they had a well-defined excitation band $\left(\lambda_{\max }=380 \mathrm{~nm}\right)$ [26]. The fluorescence quantum yield (QY) of the resultant PEI-CuNCs was $7.5 \%$ using quinine sulfate $\left(0.1 \mathrm{M} \mathrm{H}_{2} \mathrm{SO}_{4}\right.$ as solvent; $\mathrm{QY}=0.54)$ as a reference. Otherwise, the luminescence decay of the PEI-CuNCs in water was measured by a picosecond-resolved time correlated single-photon counting (TCSPC) technique (seen in Fig. S1). The decay profile was monitored at an excitation wavelength of $380 \mathrm{~nm}$, and the numerical fitting of the luminescence collected at $495 \mathrm{~nm}$ reveals time constants of $2.38 \mathrm{~ns}(37 \%)$ and $8.17 \mathrm{~ns}$ (63\%). In turn, it is good evidence of PEI-CuNCs with small size. As previously literature reported $[11,42]$, luminescence lifetimes of the few-atom metal NCs were on the order of nanoseconds, indicating that ligand-metal charge transfer was not involved in the observed size-dependent emission of NCs.

The XPS analysis was carried out to determine the oxidation state of copper in 
the PEI-CuNCs. As shown in Fig. 3, the binding energy of $\mathrm{Cu} 2 \mathrm{p}_{3 / 2}$ was observed at $932.3 \mathrm{eV}$, which was assigned to the binding energies of $\mathrm{Cu}(0)$ [22,26], and no feature of $\mathrm{Cu}$ (II) electrons was observed due to the addition of HCHO. It is of great importance to mention that the $2 \mathrm{p}_{3 / 2}$ binding energy of $\mathrm{Cu}(0)$ is only about $0.1 \mathrm{eV}$ away from that of $\mathrm{Cu}(\mathrm{I})$ [25], so it is not possible to exclude the formation of $\mathrm{Cu}(\mathrm{I})$, and the valence state of the obtained CuNCs most likely lies between 0 and +1 . The $\mathrm{C}$ 1s peak could be disintegrated into two different components at $287.8 \mathrm{eV}\left(-\mathrm{CONH}_{2}\right)$ and $284.6 \mathrm{eV}\left(-\mathrm{CH}_{2} \mathrm{CH}_{3}\right)$. The $\mathrm{N}$ 1s peaks at $398.5 \mathrm{eV}$ indicated the presence of $-\mathrm{NH}$ in PEI molecules. The peak at $531.1 \mathrm{eV}$ was in accordance with the binding energy of O 1s. Further chemical and surface properties of PEI-CuNCs were exploited by FT-IR measurements as shown in Fig. S2. Apparently, the N 1s peak can be disintegrated into two different components at $398.7 \mathrm{eV}(-\mathrm{NH})$ and $399.8 \mathrm{eV}\left(-\mathrm{NH}_{2}\right)$. Two peaks at 287.8 and $284.1 \mathrm{eV}$ were the characteristic signals of $\mathrm{C} 1 \mathrm{~s}$, which were corresponding to the $\mathrm{C}-\mathrm{O}$ and $\mathrm{C}-\mathrm{N}$ bands, respectively. The peak at $531.8 \mathrm{eV}$ was assigned to the binding energy of $\mathrm{O} 1 \mathrm{~s}$. As shown in Fig. S2, the PEI-CuNCs exhibited a number of characteristic IR bands, i.e., amide $\mathrm{C}=\mathrm{O}\left(1645 \mathrm{~cm}^{-1}\right), \mathrm{N}-\mathrm{H}\left(1570 \mathrm{~cm}^{-1}\right), \mathrm{CH}_{2}(1472$ $\mathrm{cm}^{-1}$ ) and $\mathrm{C}-\mathrm{N}\left(1309 \mathrm{~cm}^{-1}\right)$ [47]. Moreover, the broad band centered at $3280 \mathrm{~cm}^{-1}$ indicates the existence of $-\mathrm{OH}$ and $\mathrm{N}-\mathrm{H}$ bands. These results demonstrated that the CuNCs surface was enwrapped with the protection layer of PEI.

After one month preservation under ambient temperature, the resultant PEI-CuNCs still exhibit strong fluorescence and keep stable without the obvious colloidal aggregation (see Fig. S3). After irradiation by xenon lamp (450 W) with 120 min, the PL intensity of PEI-CuNCs changed slightly, showing good photostability (see in Fig. S4a). To investigate the stability of ions to potential detection, a variety of ions $\left(\mathrm{K}^{+}, \mathrm{Na}^{+}, \mathrm{Zn}^{2+}, \mathrm{Ba}^{2+}, \mathrm{Cd}^{2+}, \mathrm{Ca}^{2+}, \mathrm{Mg}^{2+}, \mathrm{Fe}^{3+}, \mathrm{Cu}^{2+}, \mathrm{Ni}^{2+}, \mathrm{Cr}^{3+}, \mathrm{Co}^{2+}, \mathrm{Mn}^{2+}\right.$ and $\left.\mathrm{CrO}_{4}{ }^{-}\right)$under identical concentration $\left(10^{-5} \mathrm{M}\right)$ were systematically introduced to the PEI-CuNCs solution $(0.05 \mathrm{mg} / \mathrm{mL})$, and the fluorescence of PEI-CuNCs were found to be quenched very rarely (see in Fig. S4b). Based on above experiments, we can conclude that the prepared PEI-CuNCs exhibited excellent colloidal stability.

\subsection{Size-dependent photoluminescent properties of CuNCs}


Interfacial synthesis has been identified as the general and effective way for preparing nanomaterials [37-39]. Noting that the structure and properties of nanomaterials should be well controlled by adjusting the interfacial factors [12], especially temperature plays an important role. By adjusting the etching temperature $\left(25,50,90\right.$ and $\left.150{ }^{\circ} \mathrm{C}\right)$, a series of PEI-CuNCs were prepared, and their TEM images are shown in Fig. 1c and Fig. S5. With the increase of the reaction temperature, the average size of NCs increases from $1.8 \mathrm{~nm}$ to $3.5 \mathrm{~nm}$. This is probably because that at lower temperature the etching reaction was relatively slower, which was beneficial for forming the smaller NCs [48]. With the increase of the temperature, the reaction became faster so that the resultant NCs tended to be larger. Owing to the quantum size effect of metal NCs, the PL intensity of NCs showed a strong dependence on their sizes $[7,49]$. As shown in Fig. 4a, with increasing the temperature form 25 to $150{ }^{\circ} \mathrm{C}$, the PL intensity of PEI-CuNCs exhibited a significant red shift $(\sim 130 \mathrm{~nm})$ from 470 $\mathrm{nm}$ (blue emission) to $600 \mathrm{~nm}$ (orange emission). Similarly, the absorption band positions in the UV-Vis spectra own red-shifts as shown in Fig. 4b. Under $365 \mathrm{~nm}$ UV irradiation, all the samples show strong emission, and the tunable color from blue to orange were clearly observed under UV irradiation (inset of Fig. 4b). The corresponding QYs of PEI-CuNCs are 8.2\%, 7.0\%, 5.3\% and 4.6\%, respectively. The results illustrate the increasing of NCs size causes the red shift of both PL and UV absorption peak, and further demonstrate the emission mechanism of these NCs follows by the particles size effect. Moreover, other two important reaction parameters for the CuNCs synthesis including the molecular weight of PEI and the amount of HCHO were also investigated, and the results are shown in Fig. S6. For comparison, normalization for PL intensity was carried out. Obviously, the emission spectra of PEI-CuNCs did no shift within the investigated window, suggesting the effect of these two factors on PL properties of the PEI-CuNCs turned out to be negligible.

\section{$3.4 \mathrm{pH}$-responsive properties of CuNCs}

As well known, the PEI can be positively charged in aqueous solution and the protonated products have a broad $\mathrm{pKa}$ values due to its three protonation levels, 
including high $\mathrm{pH}(>10)$ with PEI in its molecular form, neutral $\mathrm{pH}(\sim 7)$ with all primary amines protonated, and low $\mathrm{pH}(<4)$ with most of amines protonated $[50,51]$. Therefore, the PEI-capped nanomaterials exhibited smart $\mathrm{pH}$-responsive properties. As shown in Fig. 5a and b, the sensing action of PEI-CuNCs were characterized by fluorescence spectra in a series of buffer solutions from $\mathrm{pH} 2.0$ to 13.2. The emission peak did not shift within the investigated $\mathrm{pH}$ range. The PL intensity of PEI-CuNCs had changed a little at lower $\mathrm{pH}$ values (2.0-4.0), and increased dramatically from $\mathrm{pH}$ 5.2 to 10.1 . This is probably because that in acidic environment the interaction between the surface of PEI-CuNCs and the solution is weak, and thus the nonradiative transition is not strong, resulting in the increase of PL intensity is not obvious. At higher $\mathrm{pH}$ values (11.3-13.2), the PEI tended to chelate with CuNCs instead of protonation, favoring for the stability of CuNCs, and thus the fluorescence intensity reached the maximum value and kept constant. Quantitatively, the intensity increases approximately 20 -fold with great fluorescence at $\mathrm{pH} 13.2$. Although all the as-prepared PEI-CuNCs are colorless under visible light, they display a distinct color variation with $\mathrm{pH}$ changing under UV irradiation, such as from colorless to light blue color and finally to blue color (Fig. 5c and d). This imparts that our PEI-CuNCs, as a color indicator in $\mathrm{pH}$ detection, not only show $\mathrm{pH}$-dependent fluorescence but also display an obvious color change with increasing $\mathrm{pH}$ values, which give them an advantage over the previous used or reported nanoscale $\mathrm{pH}$-sensors [52].

\subsection{CuNCs for bioimaging and gene delivery}

The above experiments demonstrated that the PEI capped-CuNCs have some unique advantages, including good water-solubility, strong fluorescence and pH-responsive PL intensity. To be better applied in bioimaging and gene delivery, the cytotoxicity of PEI-CuNCs was evaluated through MTT assay on 293T cells. As shown in Fig. 6, the relative viabilities of $293 \mathrm{~T}$ cells treated with PEI-CuNCs still remained above $75 \%$ after $24 \mathrm{~h}$ of incubation at the concentration of $100 \mu \mathrm{g} / \mathrm{mL}$, even when the investigated conditions are much vigorous than that required for biological imaging. This indicates that the PEI-CuNCs are biocompatible and show no adverse effects to $293 \mathrm{~T}$ cells at $\mathrm{Cu}$ concentration up to $100 \mu \mathrm{g} / \mathrm{mL}$. Fig. 7 reveals the bright field, confocal fluorescent and overlay images of 293T cells incubated with 
as-prepared PEI-CuNCs for $12 \mathrm{~h}$. From the bright field image, we could see that the cells still maintain their normal morphology, thus indicating the good biocompatibility of the PEI-CuNCs in this specific dose and time point. The fluorescence image irradiated by $405 \mathrm{~nm}$ showed bright blue fluorescence within the cells, which indicated the uptake behaviors of $293 \mathrm{~T}$ cells. As reported in literature, NCs can be efficiently internalized by cells by energy-dependent, clathrin-mediated endocytosis [53]. Significantly, the fluorescent signals were distributed not only in the cytoplasm but also in the cellular nucleus, indicating that the PEI-CuNCs are readily internalized into the cell. To further improve whether the nanoclusters enter cells and where they go (seen in Fig. S7), we selected the propidium iodide to sign the cellular nucleus, and they gave out a red fluorescence. According to the overlay image, it can be clearly seen that the as-prepared PEI-CuNCs had been entered in the cellular nucleus. Taking above experimental results together it strongly suggests that our PEI-CuNCs could be act as a promising probe for optical cellular imaging, especially for labeling the oligonucleotide as a DNA probe for specific detection of nucleic acids. This result was consistent with previous reports about fluorescent NCs with similar small sizes [49].

Apart from the good biocompatibility and excellent bioimaging, the potential of as-prepared $\mathrm{PEI}-\mathrm{CuNCs}$ for gene expression in cells was further explored. Zeta potential was used to detect the surface charge of PEI-CuNCs, and the value is at $+32.5 \mathrm{eV}$ as shown in Fig. S8a, indicating the positive charge of PEI-CuNCs. As depicted in Fig. 8a, the PEI-capped CuNCs condensed DNA by electrostatic interactions between positively charged PEI on the surface of CuNCs and negatively charged phosphate backbone of plasmid DNA (pDNA) [54]. Therefore, the situation of the surface charge of the PEI-CuNCs/pDNA complexes could give insight into the ability of the cationic vectors to get access to cells [55]. Fig. S8b displays that the zeta potential data of vector/pDNA complexes, and an obvious rising trend along with an increase in weight ratio from 1 to 20 , with the maximum value of about $25 \mathrm{mV}$ was observed. As previous literature reported [56], the net positive charges of the prepared vector/DNA complexes were preferred for their intracellular uptake because of the 
interaction between the negatively charged cell membrane and the positively charged carrier/DNA complexes, which was directly correlated with efficient gene expression. For rational design of efficient gene delivery, the loading capacity of DNA as an important parameter must be taken into account, and thus the ability of PEI-CuNCs to bind plasmid DNA (pDNA) at different complex ratios was investigated by agarose gel electrophoresis assay. Fig. 8b showed that no free pDNA band could be detected when the weight ratio of PEI-CuNCs/pDNA was above 0.05 , suggesting that all the samples are capable of condensing DNA at a very low weight ratio. Based on above experiments, the as-prepared PEI-CuNCs have great potentials for gene delivery.

\section{Conclusions}

An interfacial etching approach was used to synthesize the PEI-capped CuNCs, and the etching reaction occurred at oil/water interface. Since temperature plays an important role in the interfacial synthesis, the sizes of PEI-CuNCs show a strong dependence on the temperature, and subsequently affect their PL intensity and UV absorption. The fluorescence signal of PEI-CuNCs was sensitive to the $\mathrm{pH}$ fluctuations of solution, and their PL intensity increased approximately 20 -fold with increasing the $\mathrm{pH}$ from 2.0 to 13.2. The MTT assay showed low cytotoxicity to 293T cells incorporated with the PEI-CuNCs by simple endocytosis, suggesting an excellent biocompatibility of the PEI-CuNCs. Owning to the graft with PEI, the surface of CuNCs were positively charged, and had the ability to capture DNA. In view of these attractive properties, the synthesized PEI-CuNCs are quite promising in biological applications.

\section{Acknowledgements}

This work was supported by Natural Science Foundation of Jiangsu Province, China (No. BK20140392), Natural National Science Foundation of China (No. 51502115, No. 51372265 and No. 21175060), the China Postdoctoral Science Foundation (No. 2015M581719), the Postdoctoral Research Foundation of Jiangsu Province, China (No. 1401058B), the Foundation Research Funds for the Central Universities (JUSRP51507), and the Foundation of Key Laboratory of Food Colloids and Biotechnology, Ministry of Education, Jiangnan University (No. JDST 2014-08). 


\section{References}

[1] T.H. Lee, J.I. Gonzalez, J. Zheng, R.M. Dickson, Acc. Chem. Res. 38 (2005) 534.

[2] J. Zheng, R.M. Dickson, J. Am. Chem. Soc. 124 (2002) 13982.

[3] Y.Z. Lu, W. Chen, Chem. Soc. Rev. 41 (2012) 3594.

[4] S. Choi, R.M. Dickson, J.H. Yu, Soc. Rev. 41 (2012) 1867.

[5] G. Li, R.C Jin, Acc. Chem. Res. 46 (2013) 1749.

[6] L.B. Zhang, E.K. Wang, Nano Today 9 (2014) 132.

[7] Y. Chen, H. Choi, P.V. Kamat, J. Am. Chem. Soc. 135 (2013) 8822.

[8] L. Shang, S.J. Dong, G.U. Nienhaus, Nano Today 6 (2011) 4401.

[9] Z.K. Wu, M. Wang, J. Yang, X.H. Zheng, W.Q. Cai, G.W. Meng, H.F. Qian, H.M. Wang, R.C. Jin, Small 8 (2012) 2028.

[10]Y. Yu, Z.T. Luo, Y. Yu, J.Y. Lee, J.P. Xie, ACS Nano 6 (2012) 7920.

[11]J. Zheng, C. Zhou, M.X. Yu, J.B. Liu, Nanoscale 4 (2012) 4073.

[12]D. Joseph, K.E. Geckeler, Colloids Surf. B: Biointerfaces 115 (2014) 46.

[13]A.R. Garcia, I. Rahn, S. Johnson, R. Patel, J. Guo, J. Orbulescu, M. Micic, J.D. Whyte, P. Blackwelder, R.M. Leblan, Colloids Surf. B: Biointerfaces 105 (2013) 167.

[14]L. Shang, N. Azadfar, F. Stockmar, W. Send, V. Trouillet, M. Bruns, D. Gerthsen, G.U. Nienhaus, Small 7 (2011) 2614.

[15]S.I. Tanaka, J. Miyazaki, D.K. Tiwari, T. Jin, Y. Inouye, Angew. Chem. Int. Ed. $50(2010) 431$.

[16]Y. Chen, H.P. Zhou, Y. Wang, W.Y. Li, J. Chen, Q. Lin, C. Yu, Chem. Commun. 49 (2013) 9821.

[17]C.X. Wang, Y. Wang, L. Xu, X.D. Shi, X.W. Li, X.W. Xu, H.C. Sun, B. Yang, Q. Lin, Small 9 (2013) 413.

[18]J.T. Petty, S.P. Story, J.C. Hsiang, R.M. Dickson, J. Phys. Chem. Lett. 4 (2013) 1148.

[19]T. Udayabhaskararao, Y. Sun, N. Goswami, S.K. Pal, K. Balasubramanian, T. Pradeep, Angew. Chem. Int. Ed. 51 (2012) 2155. 
[20]X.F. Jia, J. Li, L. Han, J.T. Ren, X. Yang, E.K. Wang, ACS Nano 6 (2012) 3311.

[21]S. Biswas, J.T. Miller, Y.H. Li, K. Nandakumar, C.S.S.R. Kumar, Small 8 (2012) 688.

[22]W.T. Wei, Y.Z. Lu, W. Chen, S.W. Chen, J. Am. Chem. Soc. 133 (2011) 2060.

[23]Z.H. Qing, X.X. He, D.G. He, K.M. Wang, F.Z. Xu, T.P. Qing, X. Yang, Angew. Chem. Int. Ed. 52 (2013) 9719.

[24]C. Wang, C.X. Wang, L. Xu, H. Cheng, Q. Lin, C. Zhang, Nanoscale 6 (2014) 1775 .

[25]X.F. Jia, J. Li, E.K. Wang, Small 9 (2013) 3873.

[26]N. Goswami, A. Giri, M.S. Bootharaju, P.L. Xavier, T. Pradeep, S.K. Pal, Anal. Chem. 83 (2011) 9676.

[27]X. Yuan, Z.T. Luo, Q.B. Zhang, X.H. Zhang, Y.G. Zheng, J.Y. Lee, J.P. Xie, ACS Nano 5 (2011) 8800.

[28]X.F. Jia, X. Yang, J. Li, D.Y. Li, E.K. Wang, Chem. Commun. 50 (2014) 237.

[29]A. Singh, S. Singh, S. Levcenko, T. Unold, F. Laffir, K.M. Ryan, Angew. Chem. Int. Ed. 52 (2013) 5801.

[30]L. Bao, Z.L. Zhang, Z.Q. Tian, L. Zhang, C. Liu, Y. Lin, B.P. Qi, D.W. Pang, Adv. Mater. 23 (2011) 5801.

[31]H. Li, Y.Q. Sun, L. Xu, C.X. Wang, Y.N. Jiang, Z.C. Cui, Q. Lin, RSC Adv. 3 (2013) 22849.

[32] Y.P. Gu, R. Cui, Z.L. Zhang, Z.X. Xie, D.W. Pang, J. Am. Chem. Soc. 134 (2012) 79.

[33]M.N. Martin, D.W. Li, A. Dass, S.K. Eah, Nanoscale 4 (2012) 4091.

[34]F.K. Wang, X.H. Zhang, Z. Zhang, C.B. He, J. Mater. Chem. 21 (2011) 15167.

[35] K.V. Mrudula, T.U. B. Rao, T. Pradeep, J. Mater. Chem. 19 (2009) 4335.

[36]C.N.R. Rao, K.P. Kalyanikutty, Acc. Chem. Res. 41 (2008) 489.

[37]T.U.B. Rao, T. Pradeep, Angew. Chem. Int. Ed. 49 (2010) 3925.

[38]M.A.H. Muhammed, P.K. Verma, S.K. Pal, R.C. A. Kumar, S. Paul, R.V.

Omkumar, T. Pradeep, Chem. Eur. J. 15 (2009) 10110.

[39]W.W. Guo, J.P. Yuan, E.K. Wang, Chem. Commun. 48 (2012) 3076. 
[40]D.S. Wang, Y.D. Li, Chem. Commun. 47 (2011), 47, 3604.

[41]C.X. Wang, D. Zhang, L. Xu, Y.N. Jiang, F.X. Dong, B. Yang, K. Yu, Q. Lin, Angew. Chem. Int. Ed. 50 (2011) 7587.

[42]H.W. Duan, S.M. Nie, J. Am. Chem. Soc. 129 (2007) 2412.

[43]L. Cao, X. Wang, M.J. Meziani, F.S. Lu, H.F. Wang, P.G. Luo, Y. Lin, B.A. Harruff, L.M. Veca, D. Murray, S.Y. Xie, Y.P. Sun, J. Am. Chem. Soc. 129 (2007) 11318.

[44]H.J. Lee, S.G. Lee, E.J. Oh, H.Y. Chung, S.I. Han, E.J. Kim, S.Y. Seo, H.D. Ghim, J.H. Yeum, J.H. Choi, Colloids Surf. B: Biointerfaces 88 (2011) 505.

[45]M.A.H. Muhammed, T. Pradeep, Small 7 (2011) 204.

[46]C. Wang, Y. Huang, New J. Chem. 38 (2014) 657.

[47]C.J. Liu, P. Zhang, X.Y. Zhai, F. Tian, W.C. Li, J.H. Yang, Y. Liu, H.B. Wang, W. Wang, W.G. Liu, Biomaterials 33 (2012) 3604.

[48]J. Joo, J.M. Pietryga, J.A. McGuire, S.H. Jeon, D.J. Williams, H.L. Wang, V.I. Klimov, J. Am. Chem. Soc. 131 (2009) 10620.

[49]C.X. Wang, Y. Wang, L. Xu, D. Zhang, M.X. Liu, X.W. Li, H.C. Sun, Q. Lin, B. Yang, Small 8 (2012) 3137.

[50]F. Qu, N.B. Li, H.Q. Luo, Langmuir 29 (2013) 1199.

[51]Y.Q. Dong, R.X. Wang, G.L. Li, C.Q. Chen, Y.W. Chi, G.N. Chen, Anal. Chem. 84 (2012) 6220.

[52] Y.S. Liu, D.T. Tu, H.M. Zhu, X.Y. Chen, Chem. Soc. Rev. 42 (2013) 6924.

[53]L. Yang, L. Shang, G.U. Nienhaus, Nanoscale 5 (2013) 1537.

[54]L. He, L.Z. Feng, L. Cheng, Y.M. Liu, Z.W. Li, R. Peng, Y.G. Li, L. Guo, Z. Liu, ACS Appl. Mater. Interfaces. 5 (2013) 10381.

[55]Y. Tao, Z.H. Li, E.G. Ju, J.S. Ren, X.G. Qu, Nanoscale 5 (2013) 6154.

[56]Q. Tang, B. Cao, H. Wu, G. Cheng, Langmuir 28 (2012) 16126. 


\section{Graphical abstract:}

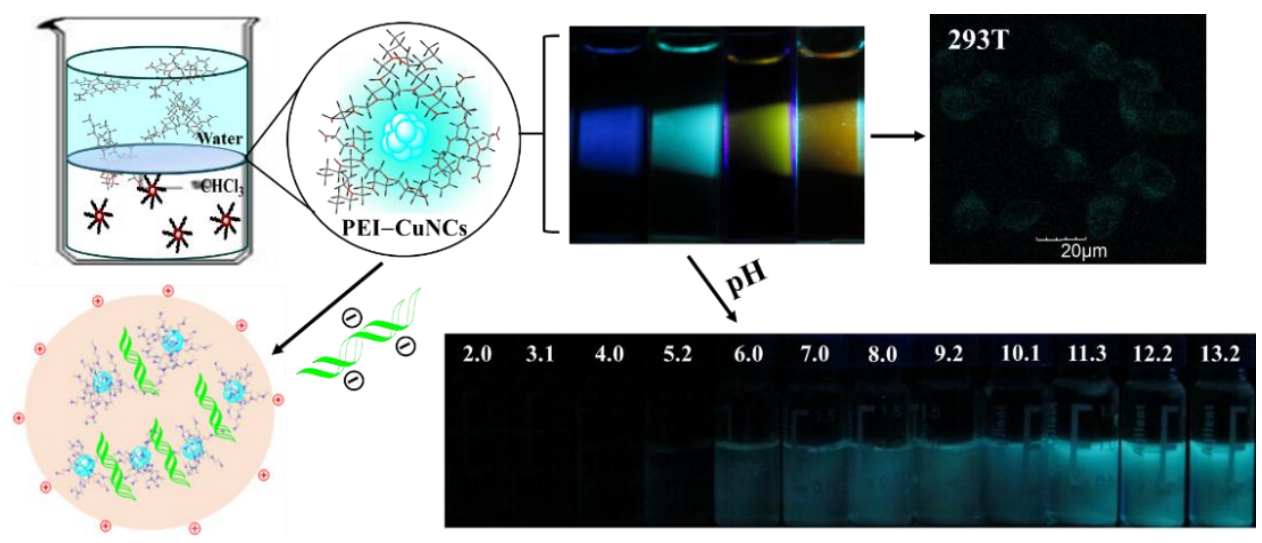




\section{Figures caption}

Scheme 1 - Schematic representation of the interfacial etching reaction of preformed CuNPs in chloroform phase with PEI in aqueous phase, and the formation of highly fluorescent and water-soluble CuNCs.

Fig. 1 - The representative TEM images (inset: size distribution) and XRD spectra of the preformed CuNPs $(a, b)$, and the synthesized CuNCs $(c, d)$.

Fig. 2 - (a) UV-Vis spectra of CuNPs in chloroform phase and CuNCs in aqueous solution; (b) Excitation $\left(\mathrm{E}_{\mathrm{X}}\right)$ and emission $\left(\mathrm{E}_{\mathrm{M}}\right)$ spectra of the resultant fluorescent PEI-CuNCs in aqueous solution (etching temperature: $50{ }^{\circ} \mathrm{C}$ ).

Fig. 3 - Typical XPS spectra of (a) Cu 2p, (b) N 1s, (c) C 1s and (d) O 1s involved in the resultant PEI-CuNCs.

Fig. 4 - (a) Tunable PL spectra of the PEI-CuNCs in aqueous solution (a significant red shift); (b) UV-Vis spectra of preformed CuNPs and resultant PEI-CuNCs prepared under different temperatures $\left(25,50,90\right.$, and $\left.150{ }^{\circ} \mathrm{C}\right)$. Inset: photographs of different PEI-CuNCs under ambient light and UV irradiation.

Fig. 5 - (a) Fluorescence spectra of PEI-CuNCs in buffers from pH 1.98 to 13.23; (b) Variation of fluorescence intensity $\left(\mathrm{E}_{\mathrm{M}}=495 \mathrm{~nm}\right)$ of PEI-CuNCs at different $\mathrm{pH}$ values; Photographs for the color changes of PEI-CuNCs in buffers at different $\mathrm{pH}$ values under visible light (c) and $365 \mathrm{~nm}$ UV light (d).

Fig. 6 - Viability of $293 \mathrm{~T}$ cells after $24 \mathrm{~h}$ incubation with different concentrations of PEI-CuNCs in the cell medium as determined by the MTT assay.

Fig. 7 - (a) Bright field, (b) confocal fluorescent and (c) overlay images of 293T cells incubating with PEI-CuNCs during emission wavelength $\lambda_{\max }$ at $500 \mathrm{~nm}$ for $12 \mathrm{~h}$. The concentration of PEI-CuNCs is $20 \mu \mathrm{g} / \mathrm{mL}$ 。

Fig. 8 - (a) Formation of PEI-CuNCs/DNA complex; (b) Agarose gel electrophoresis patterns of PEI-CuNCs/DNA complex. 

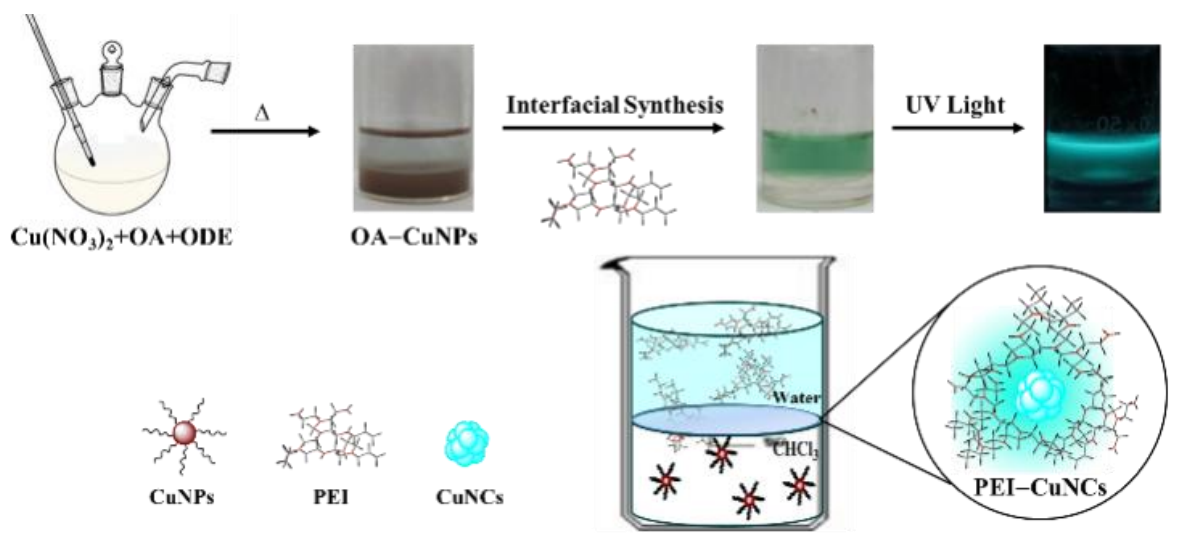

Scheme 1. Schematic representation of the interfacial etching reaction of preformed CuNPs in chloroform phase with PEI in aqueous phase, and the formation of highly fluorescent and water-soluble CuNCs.
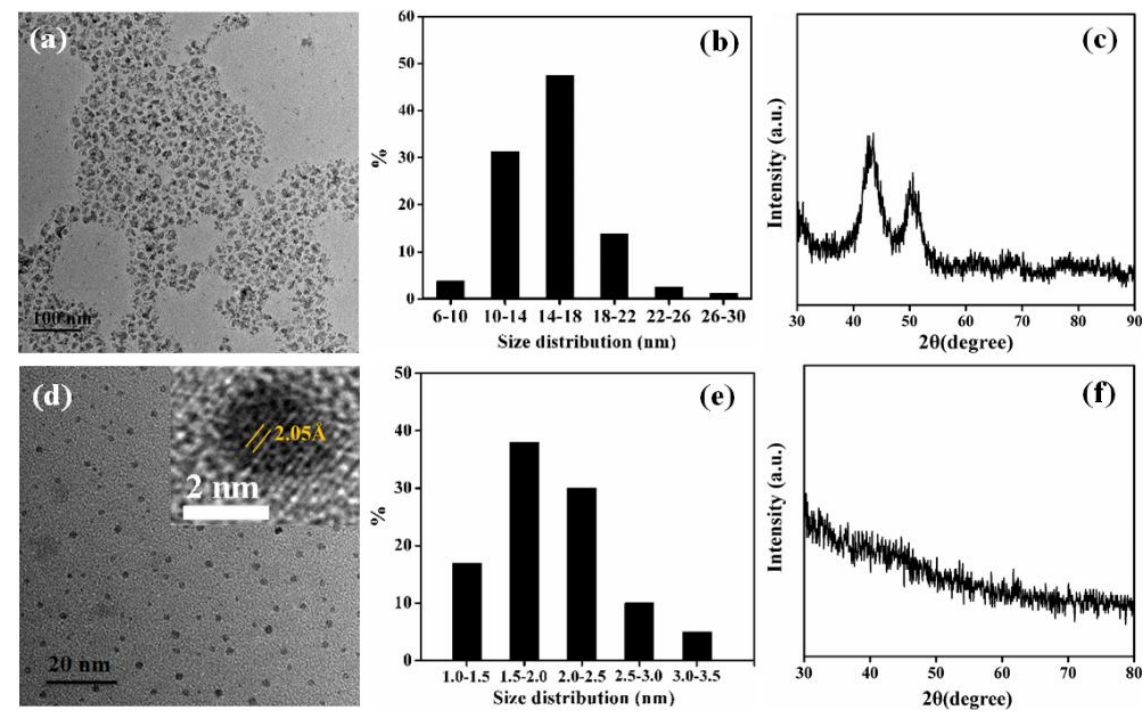

Fig. 1. The representative TEM images, size distribution and XRD spectra of the preformed CuNPs ( $a, b$ and $c$ ), and the synthesized CuNCs (d, e and f). Inset: the HRTEM image of CuNCs. 
(a)

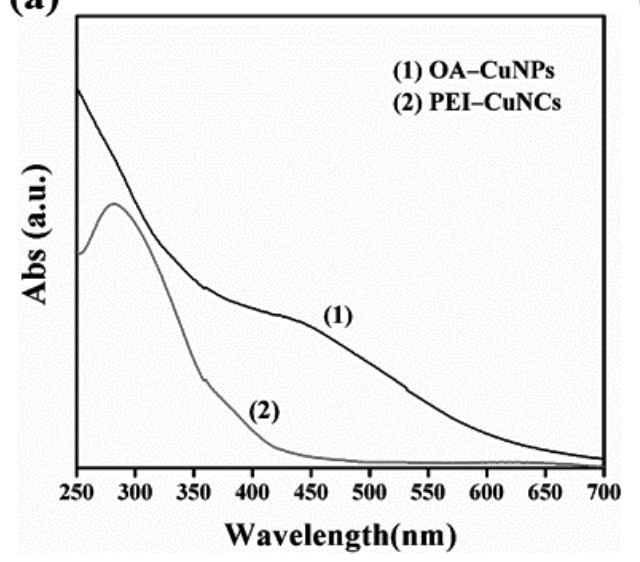

(b)

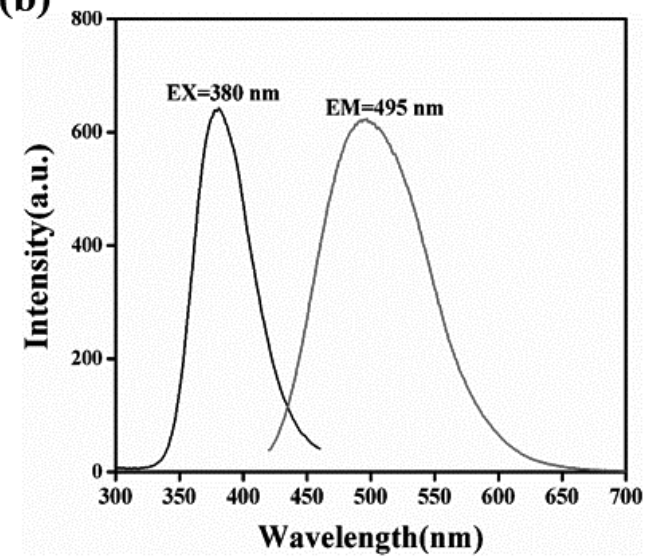

Fig. 2. (a) UV-Vis spectra of CuNPs in chloroform phase and CuNCs in aqueous solution; (b) Excitation $\left(E_{X}\right)$ and emission $\left(E_{M}\right)$ spectra of the resultant fluorescent PEI-CuNCs in aqueous solution (etching temperature: $50^{\circ} \mathrm{C}$ ).
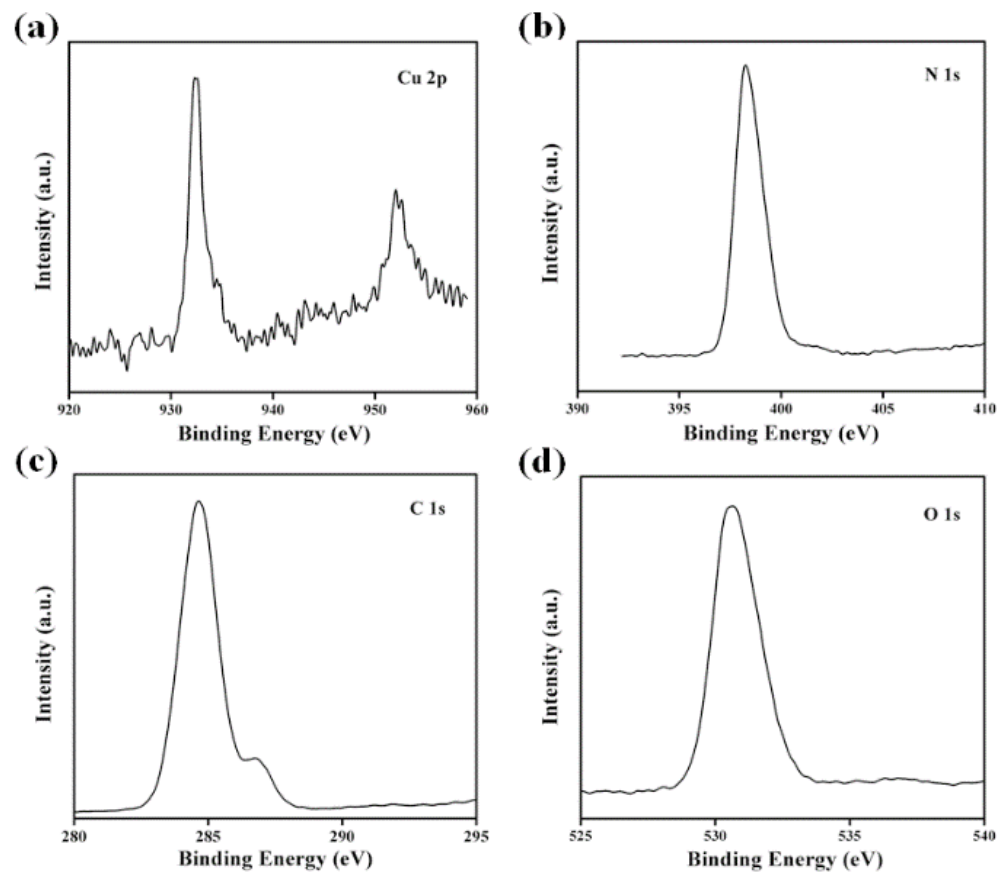

Fig. 3. Typical XPS spectra of (a) Cu 2p, (b) N 1s, (c) C 1s and (d) O 1s involved in the resultant PEI-CuNCs. 

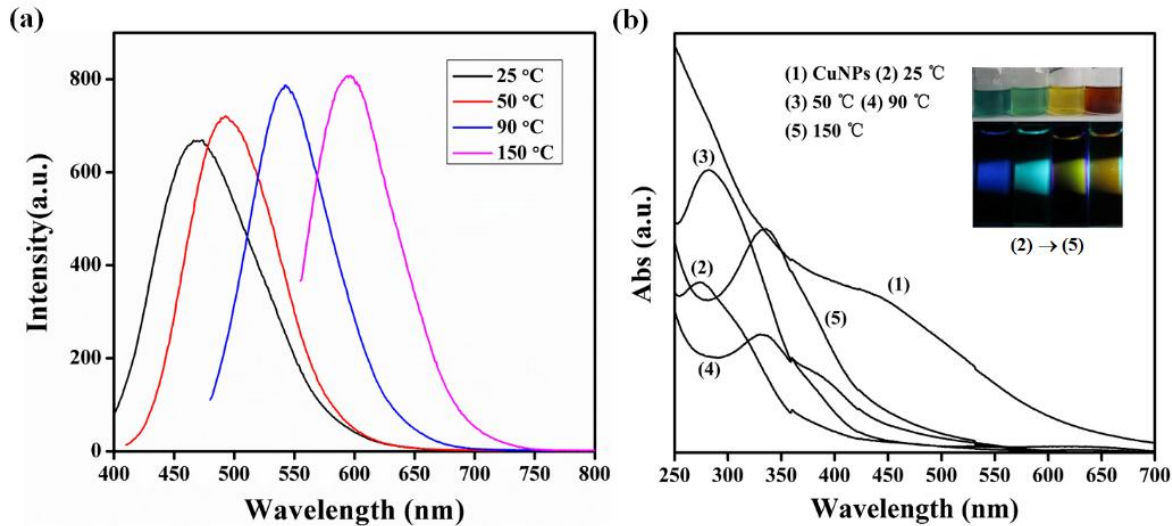

Fig. 4. (a) Tunable PL spectra of the PEI-CuNCs in aqueous solution (a significant red shift); (b) UV-Vis spectra of preformed CuNPs and resultant PEI-CuNCs prepared under different temperatures $\left(25,50,90\right.$, and $\left.150{ }^{\circ} \mathrm{C}\right)$. Inset: photographs of different PEI-CuNCs under ambient light and UV irradiation.

(a)

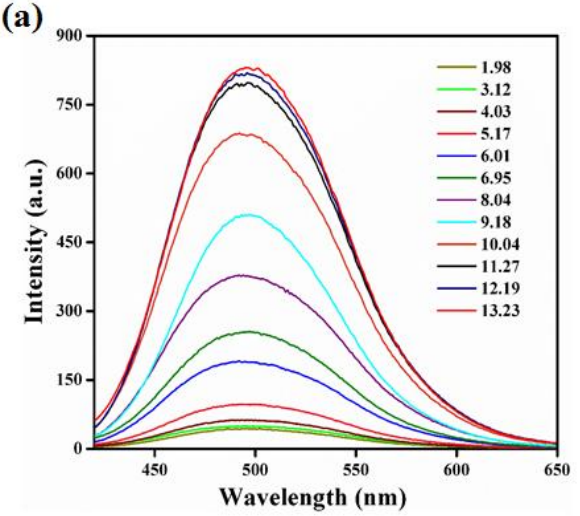

(b)

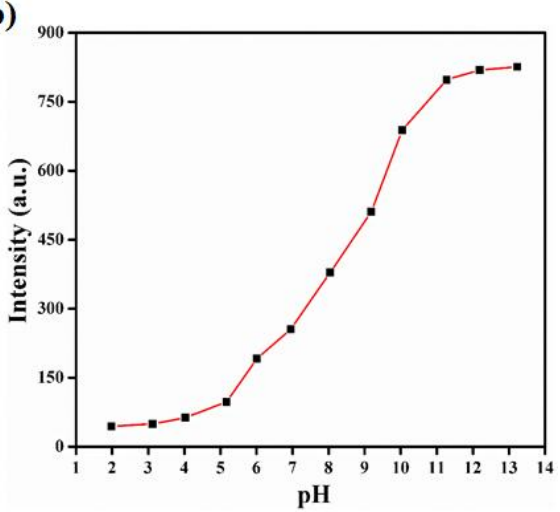

(c)

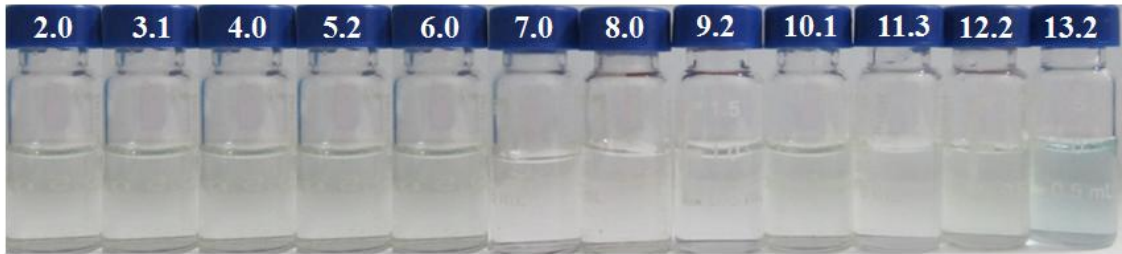

(d)

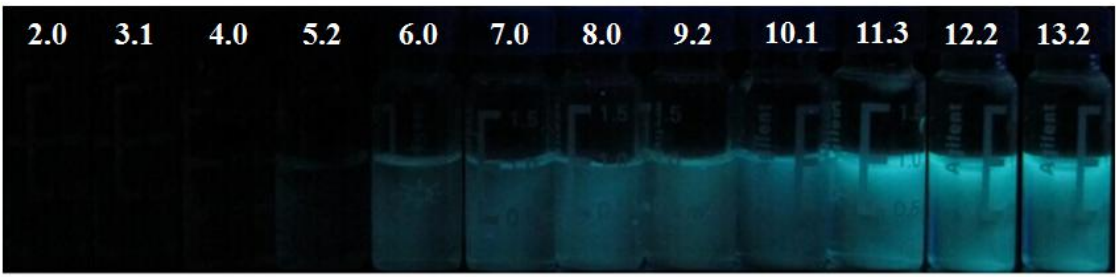

Fig. 5. (a) Fluorescence spectra of PEI-CuNCs in buffers from $\mathrm{pH} 1.98$ to 13.23; (b) Variation of fluorescence intensity $\left(\mathrm{E}_{\mathrm{M}}=495 \mathrm{~nm}\right)$ of PEI-CuNCs at different $\mathrm{pH}$ values; Photographs for the color changes of PEI-CuNCs in buffers at different $\mathrm{pH}$ values under visible light (c) and $365 \mathrm{~nm}$ UV light (d). 


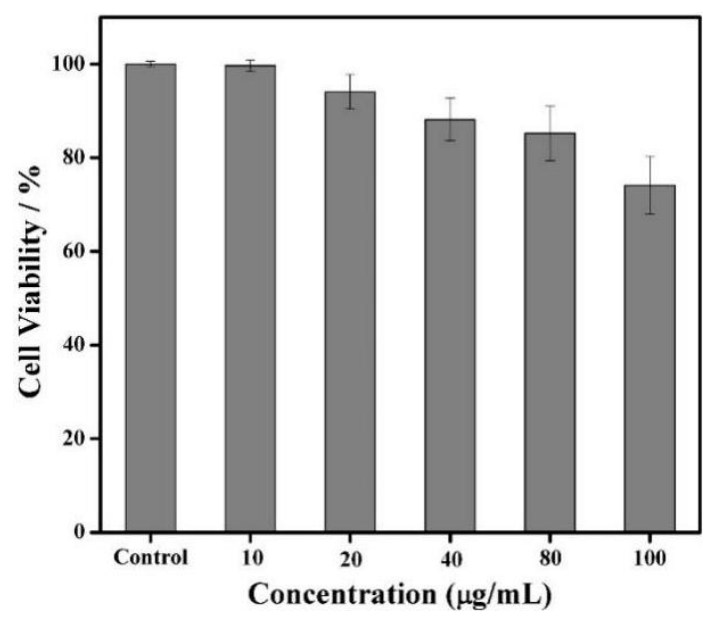

Fig. 6. Viability of $293 \mathrm{~T}$ cells after $24 \mathrm{~h}$ incubation with different concentrations of PEI-CuNCs in the cell medium as determined by the MTT assay.
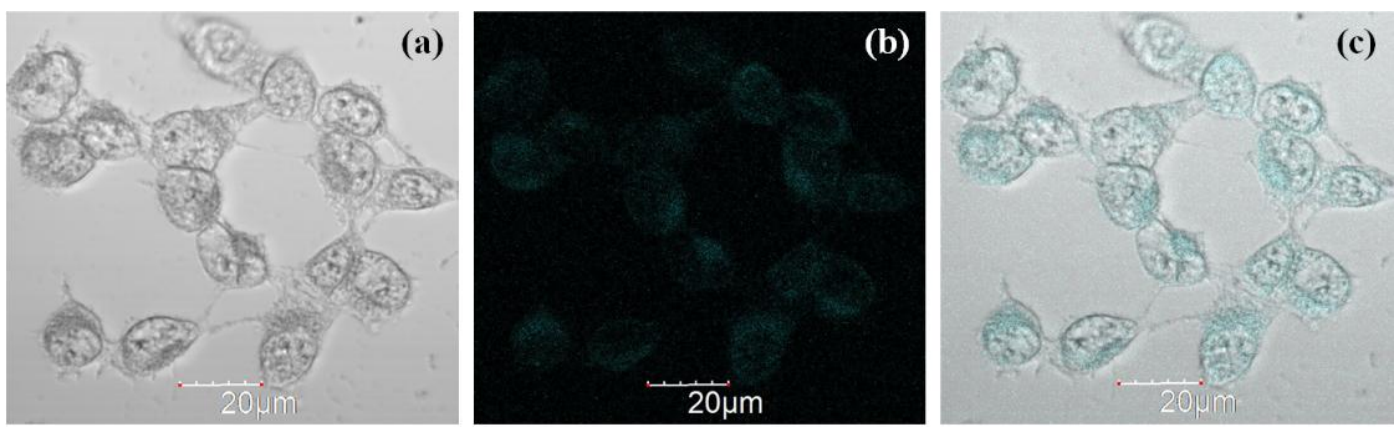

Fig. 7. (a) Bright field, (b) confocal fluorescent and (c) overlay images of 293T cells incubating with PEI-CuNCs during emission wavelength $\lambda_{\max }$ at $500 \mathrm{~nm}$ for $12 \mathrm{~h}$. The concentration of PEI-CuNCs is $20 \mu \mathrm{g} / \mathrm{mL}$. 


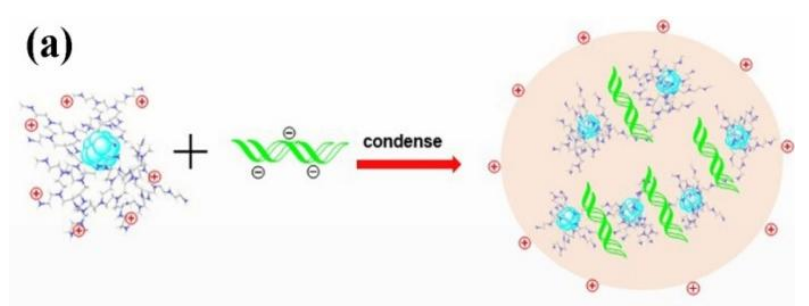

(b)

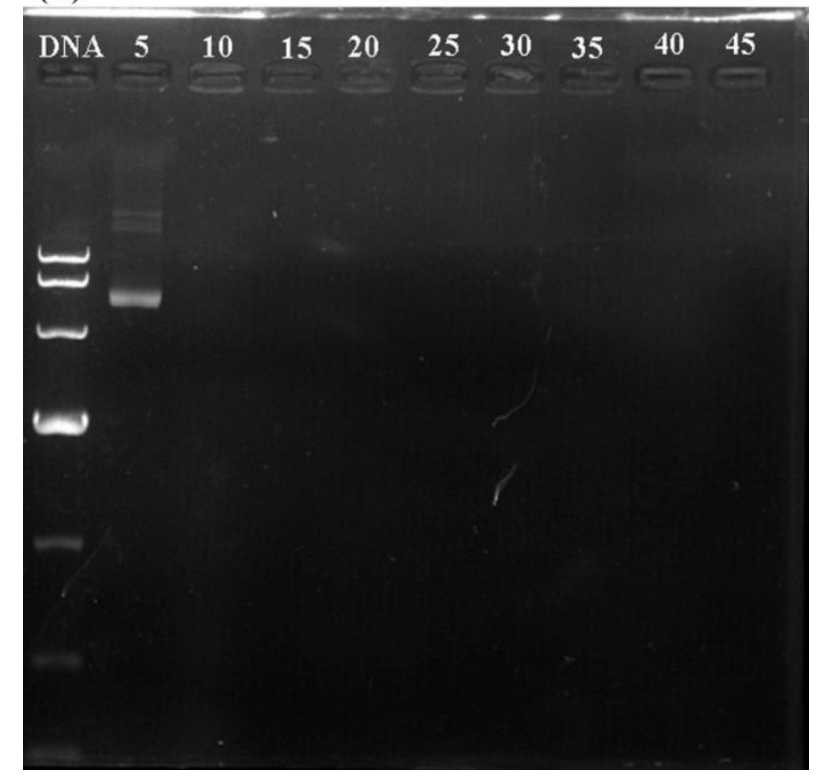

Fig. 8. (a) Formation of PEI-CuNCs/DNA complex; (b) Agarose gel electrophoresis patterns of PEI-CuNCs/DNA complex. 